In 2001, the Honor Society of Nursing, Sigma Theta Tau International and Nursing Spectrum sponsored an "Innovations in Clinical Excellence" contest to recognise exemplars of evidence-based nursing practice. The following are 2 of the winning entries.

\title{
Evidence-based development of a hospital based heart failure centre
}

Heart failure (HF) is a syndrome of shortness of breath and fatigue. It occurs when forward flow of blood out of the heart is impeded. This is the only cardiovascular condition that continues to increase in prevalence and incidence. It is a major healthcare problem, not only for the patient, but also for significant others and the community at large. The condition accounts for approximately $12-15$ million office visits and 6.5 million hospital days annually in the United States. One third of those discharged from the hospital are readmitted within 90 days because of recurrence of symptoms. The cost of HF patient care is approximately $\$ 38.1$ billion annually, draining limited resources of patients, treatment facilities, and society. ${ }^{1-3}$

\section{Type of evidence used}

To effectively deal with the problem of high HF admission rates and with the associated high number of days of hospitalisation, costs per case, and recidivism, ${ }^{14-7}$ Jersey Shore Medical Center staff used a multidisciplinary approach in developing an HF centre. Evidence was retrieved from medical, nursing, and allied health literature. Existing HF centres were observed as well.

\section{Method used to obtain and review evidence}

The project manager and a physician who strongly supported the development of an HF centre visited several model HF centres in the United States. They spoke with experts in the field and gathered many ideas. Literature was reviewed to determine a "best fit" design for the planned facility. ${ }^{4-5} 8-12$ A decision was made to have a physician directed, advanced practice nurse run centre to best serve the needs of the physicians with whom we work, and the community we treat. A decision was also made to hire an experienced advanced practice nurse (APN) with a broad cardiology background. ${ }^{13}$ The APN would run the HF programme based on guidelines developed by the project manager and medical director.

\section{Planned strategies}

The HF centre was developed with goals mutually beneficial to patients and the facility. The outpatient portion of the programme was designed to help patients retain optimal heart health outside of the hospital. APNs would run the centre, providing collaborative care and aggressive education to referred patients as indicated in the literature. ${ }^{11}{ }^{13}$ Patients would be seen on site for physical assessments and treatment as indicated in the literature. ${ }^{314-19}$ Aggressive multidisciplinary education would be offered, using the resources of the hospital to provide patients with optimal care as indicated in the literature..$^{20-23}$ Patients would be routinely telemonitored to assess level of $\mathrm{HF}$ compensation. $^{24}$

Decompensation, when diagnosed early, could be easily remedied. If patients required readmission to the hospital, they would likely be less acutely ill and remain hospitalised for a shorter time, decreasing length of stay as indicated by Bennett $e t$ $a l^{25}$ and by Rich et $a l .{ }^{26}$ The advanced practice staff would also see all hospitalised patients with HF. They were to assess patients' needs and expedite meeting those needs. The HF staff would also educate patients and provide literature for them to take home. Questions and concerns would be addressed. Diagnostic departments were to be called to ensure timely scheduling of tests. APNs were to collaborate with physicians to optimise patient care and gain referrals to the outpatient centre. Dietary consultation would be routinely requested. Case managers and social workers were given preliminary advice about potential patient discharges, so that discharge needs could be anticipated in advance of actual discharge. ${ }^{82} 27-31$

\section{How the strategies were implemented}

The APN was hired. Space was set up for the outpatient centre near a telemetry unit, within easy access of the front door. 3 rooms were provided for the HF centre: an office, an examination room, and a treatment room. Office equipment and supplies were ordered including a video system and patient literature. Hands-on teaching tools and compliance aids were ordered using funds from the Heart Failure Center Foundation. A semiprivate room is used as a treatment room. In this room, patients can rest in bed while they receive diuretics or intermittent inotropic infusion therapy. ${ }^{32}$ These patients receive care from the telemetry unit nursing staff.

Free valet parking was set up for patients. Necessary forms were designed and a registrar is on site. Many meetings were held with ancillary departments to streamline every process including medical record charting, supply distribution, consultations, information technology, reporting, and finance. Multidisciplinary meetings were held with managers from nutrition, cardiac rehabilitation, clinical pharmacy, case management, and social services departments to set up support services for outpatients. Decisions were made about how best to provide excellent customer service. Meetings were held monthly to discuss progress concerning the programme. Surveys were obtained or developed to assess patients' functional status, quality of life, and satisfaction. Such tools ${ }^{33-37}$ are administered upon enrollment in the programme, and regularly thereafter, to assess patient progress.

For the inpatient portion of the programme, patient handouts were developed. Information technology staff assisted with implementing computerised standing orders for HF patients on the coronary care, telemetry, and medical units. Additional orders were implemented including routinely prescribed medications for HF patients. Consultation guidelines were instituted for referrals to the HF centre, and nursing staff, clerical staff, physicians, and office managers attended inservice education programmes about expectations. HF guidelines were also distributed to the medical staff as recommended in the literature. $^{315}$ 


\section{Method of evaluation}

Evaluation of the inpatient portion of the programme included variables such as length of stay, cost per case, and use of $\mathrm{HF}$ medications such as angiotensin converting enzyme (ACE) inhibitors, angiotensin receptor blockers, hydralazine and nitrate combinations, and $\beta$-blockers. These variables were compared monthly and quarterly. Audits of clinical pathway variables were performed to assess effectiveness of hospitalisation. Areas of deficiency were the focus of improvement strategies through our multidisciplinary clinical service team. The HF centre's staff developed the first physician report card for our healthcare system. After a blinded audit, cardiologist variables were presented to the cardiology section, so that practice issues could be assessed, compared, and improved. Post-hospitalisation telephone surveys were conducted to assess patient satisfaction.

Evaluation for the outpatient programme included assessing quality of life and functional status (Minnesota Living with Heart Failure Questionnaire and SF 12) and physical endurance $\left(\right.$ Six-Minute Walk $\left.^{33}\right)$. These were measured upon enrollment and at regular intervals. Heart failure admission rates for our enrolled patients were compared to HF readmissions for all non-enrolled patients at 30,60, and 90 days to show the benefit of our outpatient programme. Outpatient satisfaction surveys are mailed to patients quarterly.

\section{Outcomes/results}

Hospital length of stay decreased from 8.4 to 6.5 days within 6 months. Associated cost savings to the facility were over $\$ 360000$. The first full year of operation led to continued decreases in length of stay and an additional \$255000 saved. Recidivism decreased from $33 \%$ to $13 \%$ or less

Physician report cards yielded useful information that led to awareness of prescribing practices and overall improved length of stay, cost per case, and adherence to standard HF medications. Audits of clinical pathway variables showed several areas for improvement. Resultant changes were made in areas such as (a) timely performance of 2-dimensional echocardiograms; (b) timely documentation of findings in patients' records; and (c) more accurate medical record charting for patients with renal diseases, without heart disease. These changes led to more accurate diagnostic related group (DRG) coding with reimbursement ramifications. Inpatient satisfaction surveys indicated good recall about the HF centre staff visits and the education provided.

Quality of life evaluations for outpatients showed a 33\% improvement in functional status and physical endurance, and a $25 \%$ improvement in quality of life during the first 6 months of participation with the centre. Use of ACE inhibitors was found to be better for outpatients at the centre than for inpatients$89 \%$ v 50\%, respectively. Patient satisfaction with the programme remains high. Patients and family members have written letters of praise and support. Some have even contributed to the hospital's Heart Failure Center Foundation.

\section{Lessons Learnt}

Development of the HF centre was challenging. Several changes were made in the nursing care process for patients receiving intravenous inotropic infusions and diuretic therapy. Physician report cards were amended for enhanced accuracy. The clinical pathway has evolved into a process, rather than a form to be completed. Physician satisfaction surveys are currently being developed.

This hospital based, APN administered heart failure centre is an effective way to decrease length of stay and associated financial burdens to patients, our facility, and ultimately, to society.
Outpatients enrolled in this programme greatly benefit from a decrease in recidivism and from improved functional status, physical endurance, and quality of life.

MARYANNE CROWTHER, RN, MSN, APNC, CCRN ANN MAROULIS, RN, MSN, APNC, CCRN

NANCY SHAFER-WINTER, RN, MSN, APNC, CCRN, CNA, CPHQ RICHARD HADER, RN, PhD, CHE, CNA, CPHQ Jersey Shore Medical Center Neptune, New Jersey, USA

Reprinted with permission of the Honor Society of Nursing, Sigma Theta Tau International from Crowther M, Maroulis A, Shafer-Winter, et al. Evidence-based development of a hospitalbased heart failure center. Online J Knowl Synth Nurs 2002;9:5C

1 American Health Consultants. Congestive heart failure: the disease state management resource. Vol 2. Atlanta: American Health Consultants, 1998.

2 Hunt SA, Baker DW, Chin MH, et al. ACC/AHA guidelines for the evaluation and management of chronic heart failure in the adult:executive summary: a report of the American College of Cardiology/American Heart Task Force on Practice Guidelines. J Am Coll Cardiol 2001;38:2101-13. http.//www.acc.org/ clinical/guidelines/failure/exec_sum/hf_index.htm

3 Consensus recommendations for the management of chronic heart failure. On behalf of the membership of the Advisory Council to Improve Outcomes Nationwide in Heart Failure. Am J Cardiol 1999:83:1A-38A.

4 Brass-Mynderse NJ. Disease management for chronic congestive heart failure.J Cardiovasc Nurs 1996;11:54-62.

5 Cardiology Preeminence Roundtable. Beyond four walls: cost-effective management of chronic congestive heart failure. Washington, DC: Advisory Board Company, 1994

6 Knox D, Mischke L. Implementing a congestive heart failure disease management program to decrease length of stay and cost. J Cardiovasc Nurs 1999;14:55-74.

7 Wells N, Johnson R, Salyer S. Interdisciplinary collaboration. Clin Nurse Spec 1998;12:161-8.

8 Brubakken KM, Janssen WR, Ruppel DL. CNS roles in implementation of a differentiated case management model. Clin Nurse Spec 1994;8:69-73.

9 Chapman DB, Torpy J. Development of a heart failure center: a medical center and cardiology practice join forces to improve care and reduce costs. Am J Manag Care 1997;3:431-7.

10 Fonarow GC, Stevenson LW, Walden JA, et al. Impact of a comprehensive heart failure management program on hospital readmission and functional status of patients with advanced heart failure. J Am Coll Cardiol 1997;30:725-32.

11 McCormick SA. Advanced practice nursing for congestive heart failure. Crit Care Nurs Q 1999;21:1-8.

12 Wilkes RM, Dukes KR, Feagles LL, et al. Kaiser Permanente's approach to congestive heart failure in south San Francisco. Journal of Clinical Outcomes Management 1999;6:37-40.

13 Paul S. Implementing an outpatient congestive heart failure clinic: the nurse practitioner role. Heart Lung 1997;26:486-91.

14 Effects of enalapril on mortality in severe congestive heart failure. Results of the Cooperative North Scandinavian Enalapril Survival Study of the Cooperative North Scandinavian Enalapril Survival Study
(CONSENSUS). The CONSENSUS Trial Study Group. $N$ Engl J Med 1987:316:1499-35.

15 Heart Failure Society of America (HFSA) practice guidelines. HFSA guidelines for management of patients with heart failure caused by left guidelines for management of patients with heart failure caused by left
ventricular systolic dysfunction-pharmacological approaches. J Card Fail ventricular systo $1999 ; 5: 357-82$.
199.

16 Hosenpud JD, Greenberg BH, editors. Congestive heart failure: pathophysiology, diagnosis, and comprehensive approach to management. New York: pringer-Verlag, 1994.

17 Kramer BK, Schweda F, Riegger GA. Diuretic treatment and diuretic resistance in heart failure. Am J Med 1999;106:90-6.

18 Effect of enalapril on survival in patients with reduced left ventricular ejection fractions and congestive heart failure. The SOLVD Investigators. $N$ Engl J Med 1991;325:293-302.

19 Stevenson LW, Massie BM, Francis GS. Optimizing therapy for complex or refractory heart failure: a management algorithm. Am Heart $J$ 998;135:S293-309.

20 Allison M, Keller C. Physical activity in the elderly: benefits and intervention strategies. Nurse Pract 1997;22:53-4, 56, 58 passim.

21 Kostis JB, Rosen RC, Cosgrove NM, et al. Nonpharmacologic therapy improves functional and emotional status in congestive heart failure. Chest 1994;106:996-1001.

22 Luniewski M, Reigle J, White B. Card sort: an assessment tool for the educational needs of patients with heart failure. Am J Crit Care 1999;8:297-302.

3 Simon-Weinstein M. Cardiac rehabilitation: nonpharmacologic treatment for congestive heart failure. J Am Acad Nurse Pract 1999;11:293-6.

24 Johnson MJ, Frank DI. Effectiveness of a telephone intervention in reducing anxiety of families of patients in an intensive care unit. Appl Nurs Res 1995;8:42-3.

25 Bennett SJ, Huster GA, Baker SL, et al. Characterization of the precipitants of hospitalization for heart failure decompensation. Am J Crit Care 1998;7:168-74.

26 Rich MW, Vinson JM, Sperry JC, et al. Prevention of readmission in elderly patients with congestive heart failure: results of a prospective randomized pilot study. J Gen Intern Med 1993;8:585-90.

27 Ethridge P, Lamb GS. Professional nursing case management improves quality, access and costs. Nurs Manage 1989;20:30-5.

28 Haddock KS. Collaborative discharge planning: nursing and social services. Clin Nurse Spec 1994:8:248-59, 288 .

29 Krumholz HM, Butler J, Miller J, et al. Prognostic importance of emotional support for elderly patients hospitalized with heart failure. Circulation 7:958-64.

30 Lynn-McHale DJ, Fitzpatrick ER, Shaffer RB. Case management: development of a model. Clin Nurse Spec 1993;7:299-307. 
31 Philbin EF, Rocco TA, Lindenmuth NW, et al. The results of a randomized trial of a quality improvement intervention in the care of patients with 32 Patel MB, Kaplan IV, Patni RN, et al. Sustained improvement in flow-mediated vasodilation after short-term administration of dobutamine flow-mediated vasodilation after short-term administration of dobutamin in patients with severe congestive heart failure. Circulation 1999;99:60-4. Lucas C, Stevenson LW, Johnson W, et al. The 6-min walk and peak oxygen Heart J 1999;138:618-24.
34 Bittner V. Determining prognosis in congestive heart failure: role of the 6-minute walk test. Am Heart J 1999;138:593-6.

5 Dracup K Walden JA Stevenson LW, et al Quality of life in patients with advanced heart failure. J Heart Lung Transplant 1992;1 1:273-9.

36 Hawthorne MH, Hixon ME. Functional status, mood disturbance and quality of life in patients with heart failure. Prog Cardiovasc Nurs 1994:9:22-32. quality of life in patients with heart failure. Prog Cardiovasc Nurs 1994;9:22-32.

Woo MA, Moser DK, Stevenson LW, et al. Six minute walk test and heart rate variability: lack of association in advanced stages of heart failure. Am J
Crit Care $1997: 6: 348-54$

\section{Using a FOCUS-PDCA quality improvement model for applying the severe traumatic brain injury guidelines to practice: process and outcomes}

Trauma teams strive to provide care based on best practice. Exploring the clinical outcomes of patients sustaining severe traumatic brain injury (TBI) at our trauma centre from 1994-97 we found that the outcomes were marginal at best: $43 \%$ of our patients expired and 30\% suffered severe disability. These results were consistent with those of some studies on TBI published in the 1980s. Researchers in the past decade have used new technology for monitoring the effects of secondary brain injury and examined the effects of various treatment modalities on the outcomes of patients with TBI. In 1995, the American Association of Neurological Surgeons (AANS) evidence-based clinical guidelines for managing severe TBI were published. ${ }^{1}$ The guidelines recommended changes in the care of patients with TBI and challenged caregivers to evaluate their practices and examine the clinical outcomes of this high risk group.

Motivated by the new practice recommendations and the potential for greatly affecting patient outcomes, Mission Hospital Regional Medical Center's (MHRMC) multidisciplinary neurotrauma team convened in 1997 to begin performance improvement. Current practice was examined and new hospital based clinical guidelines were developed. Numerous changes were recommended as the team dismantled current practice patterns and constructed new care priorities. The result was a series of algorithms with established outcomes at every phase of the patient's hospital course. Four years after integrating the changes in practice, the team evaluated prospectively collected data to determine outcomes for patients with severe TBI. Current outcomes (2001 data) indicated that $72.8 \%$ of patients had a good outcome (no disability to moderate disability), $13.6 \%$ had severe disability to persistent vegetative state, and $13.6 \%$ died. We will present a FOCUS-PDCA performance improvement approach to show the processes used to apply national scientific guidelines to the clinical setting. Statistical analysis using an ordinal regression model will show outcome data emphasising the positive aspects of applying evidence-based guidelines to practice.

\section{Summary of FOCUS-PDCA}

FOCUS is an acronym for the words find, organise, clarify, understand, and select. PDCA is an acronym for plan, do, act, and check results.

\section{FIND A PROCESS TO IMPROVE}

The trauma/neurosurgical physician and nursing leaders at MHRMC gathered in 1997 to facilitate the transition of research based scientific guidelines to clinical practice. The guidelines for managing severe head injury developed by the AANS challenged physicians and other health team members to examine current practice regarding severe TBI patients and to alter care practices, which had been viewed as the "gold standard" of care for years. Analysis of the TBI guidelines and related literature indicated recommendations to avoid hypoxia and hypotension; maintain a mean arterial pressure $<90 \mathrm{~mm}$ $\mathrm{Hg}$ and cerebral perfusion pressure $>70 \mathrm{~mm} \mathrm{Hg}$ using fluids and vasopressors; treat intracranial pressure (ICP) > $20 \mathrm{~mm} \mathrm{Hg}$; avoid hyperventilation to control ICP unless cerebral oxygenation measured; give boluses of mannitol intermittently and replace fluids to maintain euvolemia; use barbiturates for uncontrollable ICP; decrease stimulation in the environment; treat fever aggressively; use sedation or analgesia in continuous modes; and incorporate clinical pathways and algorithms concerning planned therapy including weaning procedures. ${ }^{1}$

\section{ORGANISE TO IMPROVE THE PROCESS}

The team leaders began by examining the current care processes for the population with TBI at MHRMC. They conducted a review of the literature and contacted a manufacturer of cerebral oxygenation $\left(\mathrm{SjO}_{2}\right)$ catheters concerning product availability. A decision was made by the leaders to form a multidisciplinary team to examine current practice, analyse the published TBI guidelines and research, and develop new treatment plans.

\section{CLARIFY THE ISSUE}

The neuro clinical nurse specialist (CNS) conducted a retrospective chart review of the previous 3.5 years. Patients included in the study were those with a closed head injury and (a) Glasgow Coma Score (GCS) from 3-8, (b) abnormal computed tomography (CT) scan of the brain, (c) age $>8$ years, and (d) ICP monitoring. Excluded from the study results were patients with penetrating head injury; patients who died or were pronounced brain dead within 24 hours of admission; patients with a GCS 3-8 because of alcohol, seizures, or systemic injury; and patients with absence of head injury as determined by CT or clinical exam. In all, 1937 trauma records were reviewed (January 1994 to June 1997). Almost half of the patients had sustained some form of head injury but only 37 met inclusion criteria. Results of the outcome study were that $27 \%$ had an outcome of zero to moderate disability; $30 \%$ had severe disability or persistent vegetative state (PVS); and 43\% died.

After the chart audit, a multidisciplinary task force was established consisting of trauma surgeons, neurosurgeons, anaesthesiologists, intensivists, rehabilitation personnel, critical care nurses, respiratory therapists, and pharmacists. Before the meeting, all members received a copy of the TBI guidelines and supporting research articles. The neuro CNS facilitated 2 meetings to compare the guidelines to current practice and establish clinical guidelines for use at the hospital. 
UNDERSTAND THE SOURCES OF VARIATION AND SELECT THE PROCESS TO IMPROVE

The team discovered that current practice deviated from the published guidelines in several areas including blood pressure (BP) and fluid management; use of hyperventilation to treat increased ICP; use of medications to decrease cerebral oxygen demand, enhance BP, and decrease ICP; temperature management; and use of technology such as $\mathrm{SjO}_{2}$ catheters to monitor cerebral oxygenation. Recommendations from members of the team were to discontinue the old treatment processes and implement a new care process incorporating the AANS TBI guidelines and recommendations in the literature that were focused on decreasing ICP, maximising BP, and optimising cerebral oxygenation. The team integrated information on manipulation of cerebral oxygenation so as to maintain normal oxygen levels. ${ }^{2}$

\section{PLAN, DO, AND ACT-JUNE 1997}

Changes were recommended as the team changed practice patterns and implemented new care priorities. Every aspect of caring for patients with severe TBI from admission in the emergency department (ED), through the operative and intensive care unit (ICU) phase, to rehabilitation was examined. The result was to develop a series of algorithms with established outcomes at every phase of a patient's hospital course. Input from every member of the multidisciplinary team was sought for the care process. $\mathrm{SjO}_{2}$ catheters were stocked in the hospital, a procedure was developed, and the staff was educated. The team developed a TBI documentation form, algorithms for the ICU team, and clinical guidelines addressing the needs of adults and children. In addition, staff members in the paediatric and adult ICUs, ED, and operating room (OR) were educated about the new process. The first patient to be cared for using the new guidelines was admitted in June 1997. This change in practice required close supervision and clinical support of team members by the neuro CNS who provided clinical support for all shifts.

\section{CHECK RESULTS-JUNE 1998}

Analysis of care processes, data, and outcomes was integral to maintain consistency of care and coordination of services. Data were collected prospectively by the neuro CNS. Analysing outcomes for 18 patients using the new guidelines indicated that $61 \%$ had a good outcome with zero to moderate disability; $11 \%$ had severe disability or PVS; and 27\% died. Feedback from members of the team included several concerns regarding the management of these patients.

Team members were concerned about variations in intubation procedures; variation in maintaining minimum BP levels for the ED and OR phases of care; variation in timing the placement of $\mathrm{SjO}_{2}$ catheters; variations in intra-operative management; and instances of increased incidence of pneumonia, poor renal perfusion, nutritional problems, and acute withdrawal symptoms because of sudden removal of analgesics.

\section{ACT-August 1998}

The neuro CNS reconvened the multidisciplinary team and reviewed the results of the first year. The key issues identified above were divided into phases of care and changes in practice were recommended. New clinical algorithms were developed. The team developed (a) protocols for rapid sequence intubations, narcotic withdrawal, and vasopressor support; (b) goals such as BP, ICP, and cerebral oxygenation levels for each phase of care; (c) nutritional management goals, with emphasis on post-pyloric feedings, which would provide nutrition into the small intestine instead of into the stomach; (d) interventions for aggressive pulmonary management; and (e) ways to use nursing research about management of environmental stimulation and family presence. We implemented changes in practice after approval by committees and staff education.

\section{CHECK AGAIN-FEBRUARY 2000}

Outcome data were evaluated once again. 56 patients were cared for since the change in practice in June 1997 and dramatic improvement in outcomes was noted: $69 \%$ had a good outcome of zero to moderate disability, 14\% had severe disability or PVS, and $16 \%$ died. We sought the assistance of an independent statistician to analyse the data. Patients in Group $1(n=37)$ were those treated before the TBI guidelines were implemented and patients in Group $2(n=56)$ were those treated after TBI guidelines were implemented. A comparison of descriptive statistics using a student $t$ test revealed no significant difference between the 2 groups concerning the variables of age, injury severity score, GCS on admission, number of days receiving ICP monitoring, number of days on mechanical ventilation, ICU length of stay (LOS), and hospital LOS. Statistically significant differences were noted between the 2 groups concerning hospital charges (\$197 128 average for patients in Group 1 and \$293 065 average for patients in Group 2). Using an ordinal regression model, the odds for significant variables were examined and adjusted for the effect of all other variables in the model. Results of the ordinal regression model were (a) patients in Group 2 had odds of a good outcome relative to odds of a poor outcome or death 9 times higher compared with patients in Group $1(\mathrm{p}=0.005)$; (b) patients with a GCS $>8$ at the time of admission had odds of a good outcome 6.58 times higher compared with the patients admitted with a GCS $<8(\mathrm{p}=0.003)$; and (c) odds of a good outcome decreased by a factor of 0.92 for each 1 year increase in patients' age starting at age $9(\mathrm{p}=0.0005)$.

Team members believe the change in outcomes of the severe TBI population was because of an aggressive standardised approach to managing multiple parameters and to the integration of advanced technology related to brain oxygenation. By integrating team interventions into one protocol, nurses, physicians, respiratory therapists, and members of other disciplines coordinated their interventions and understood the effect on patients. Using a standardised approach has been reported to be successful in other centres. ${ }^{3-5}$

\section{ACT AGAIN-200O}

The team recognised the need to encourage more family involvement in the care of patients with severe TBI. The TBI Task Force 2000 met and included hospital staff from all units, 4 former patients, and several family members. Using a qualitative approach, input from all members was gathered and 4 teams established new care practices involving: (a) patients emerging from coma; (b) structure of the physical environment in all units; (c) consistency and continuity of care; and (d) patient and family education and support.

Families are educated about the ICU environment and encouraged to be present at their ICU patient's bedside as much as possible. Therapeutic touch by family members is encouraged and supported. Members of the nursing and rehabilitation teams from units where the patient will be cared for meet the family while the patient is in the ICU. Families receive relevant education as patients move through each phase of care. Care practices and outcomes are continually monitored.

Data collected about patients $(n=81)$ at the end of fiscal year 2000 showed that $72.8 \%$ had good outcomes of zero to moderate disability, $13.6 \%$ had severe disability or PVS, and $13.6 \%$ died. These outcomes indicated to the team that the changes in practice and intense cooperation resulted in a difference in their 
patients. Each team member's collaboration and respect for others were evident in actions and words. This multidisciplinary team evolved and became synergistic with each patient and family. This synergy has enabled us to affect outcomes of severely brain injured patients at MHRMC.

\section{MARY KAY BADER, RN, MSN, CCRN, CNRN \\ SYLVAIN PALMER, MD, FACS \\ CONNIE STALCUP, RN, MSN \\ THOMAS SHAVER, MD, FACS \\ Mission Hospital Regional Medical Center \\ Mission Viejo, California, USA}

Reprinted with permission of the Honor Society of Nursing, Sigma Theta Tau International from Bader MK, Palmer S, Stalcup C, et al. Using a FOCUS-PDCA quality improvement model for applying the severe traumatic brain injury guidelines to practice: process and outcomes. Online J Knowl Synth Nurs 2002;9:4C.

1 Bullock R, Chestnut R, Clifton G, et al. Guidelines for the management of severe brain injury. New York: Brain Trauma Foundation/American Association of Neurological Surgeons, 2000 .

2 Robertson CS, Valadka AB, Hannay HJ. Prevention of secondary ischemic insults after severe head injury. Crit Care Med 1999;27:2086-95.

3 Baethmann A, Lehr D, Wirth A. Prospective analysis of patient management in severe head injury. Acta Neurochir Suppl (Wien)

4 McKinley BA, Parmley CL, Tonneson AS. Standardized management of intracranial pressure: a preliminary clinical trial.J Trauma 1999;46:271-9. 5 Simons R, Eliopoulos V, Laflamme D, et al. Impact on process of trauma care delivery one year after the introduction of a trauma program in a provincial trauma center. J Trauma 1999;46:811-6.

\section{Acknowledgements}

A s our sixth year of publication begins, we would like to thank the many people who have contributed their time, expertise, - and enthusiasm to Evidence-Based Nursing over the past year.

Our Associate Editors (listed on the inside front cover), who helped us to select articles that are clinically important and relevant to practising nurses, as well as methodologically sound; reviewed and edited numerous structured abstracts and commentaries; and so much more.

ur panel of advisers on qualitative research, who helped us to select qualitative research papers for abstraction:

Wally Barr, Liverpool, UK

Caroline Carlisle, Liverpool, UK

Chantal Caron, Sherbrooke, Quebec, Canada

Marlene Z Cohen, Houston, Texas, USA

Heather MacDonald, Fredericton, New Brunswick, Canada

Jenny Ploeg, Hamilton, Ontario, Canada
Rita Schreiber, Victoria, British Columbia, Canada

Cynthia Russell, Memphis, Tennessee, USA

Carl Thompson, York, UK

Sally Thorne, British Columbia, Canada

Carol Tishelman, Stockholm, Sweden

The individuals who helped us in the selection of quantitative studies that are clinically important and relevant to practising nurses:

Brenda Morgan, London, Ontario, Canada

Jennifer Skelly, Hamilton, Ontario, Canada

Brian Hutchison and Trevor Sheldon, who provided thoughtful methodological advice about selected studies.

The people who served as peer reviewers for the EBN Notebook and Implementation Forum, for their thoughtful and timely feedback:

Kate Flemming, York, UK

Pat Mandy, Hamilton, Ontario, Canada

Dorothy McCaughan, York, UK

David Torgerson, York, UK

Carl Thompson, York, UK

\section{Correction}

In the October 2002 issue, the authors of the EBN users' guide (Evaluation of studies of health economics) were listed incorrectly. They should have been listed as follows:

Patricia W Stone, RN, PhD

Columbia University, New York, New York, USA

Christine R Curran, RN, PhD, CNA

The Ohio State University and Medical Center, Columbus, Ohio, USA

Suzanne Bakken, RN, DNSc, FAAN

Columbia University, New York, New York, USA

Patricia H Walker, RN, PhD, FAAN

Graduate School of Nursing, Uniformed Services University of the Health Sciences, Bethesda, Maryland, USA 